\title{
Effects of coffee and caffeine anhydrous on strength and sprint performance
}

\author{
ERIC T. TREXLER ${ }^{1}$, ABBIE E. SMITH-RYAN ${ }^{1}$, ERICA J. ROELOFS ${ }^{1}$, KATIE R. HIRSCH ${ }^{1}$, and \\ MEREDITH G. MOCK ${ }^{1}$ \\ ERIC T. TREXLER: trexlere@live.unc.edu; ERICA J. ROELOFS: eroelofs@live.unc.edu; KATIE R. HIRSCH: \\ ktrose23@live.unc.edu; MEREDITH G. MOCK: meremock@live.unc.edu \\ ${ }^{1}$ Applied Physiology Laboratory, Department of Exercise and Sport Science, University of North \\ Carolina, Chapel Hill, NC, USA
}

\section{Abstract}

Caffeine and coffee are widely used among active individuals to enhance performance. The purpose of the current study was to compare the effects of acute coffee (COF) and caffeine anhydrous (CAF) intake on strength and sprint performance. Fifty-four resistance-trained males completed strength testing, consisting of one-rep max (1RM) and repetitions to fatigue (RTF) at $80 \%$ of 1 RM for leg press (LP) and bench press (BP). Participants then completed five, ten-second cycle ergometer sprints separated by one minute of rest. Peak power (PP) and total work (TW) were recorded for each sprint. At least 48 hours later, participants returned and ingested a beverage containing CAF (300 mg flat dose; yielding 3-5 mg/kg bodyweight), COF ( $8.9 \mathrm{~g} ; 303 \mathrm{mg}$ caffeine), or placebo (PLA; $3.8 \mathrm{~g}$ noncaloric flavoring) 30 minutes before testing. LP 1RM was improved more by COF than CAF ( $\mathrm{p}=0.04)$, but not PLA $(\mathrm{p}=0.99)$. Significant interactions were not observed for BP 1RM, BP RTF, or LP RTF ( $>>0.05)$. There were no sprint $\times$ treatment interactions for PP or TW ( $p>0.05$ ). 95\% confidence intervals revealed a significant improvement in sprint 1 TW for CAF, but not COF or PLA. For PLA, significant reductions were observed in sprint $4 \mathrm{PP}$, sprint $2 \mathrm{TW}$, sprint $4 \mathrm{TW}$, and average TW; significant reductions were not observed with CAF or COF. Neither COF nor CAF improved strength outcomes more than PLA, while both groups attenuated sprint power reductions to a similar degree. Coffee and caffeine anhydrous may be considered suitable pre-exercise caffeine sources for high-intensity exercise.

\section{Keywords}

Ergogenic aids; high-intensity exercise; anaerobic exercise; resistance training; maximal strength

\section{Introduction}

Caffeine is the most widely consumed psychoactive substance in the world, with estimates of average daily intakes as high as $4 \mathrm{mg} / \mathrm{kg}$ of bodyweight in US adults (Barone \& Roberts, 1996). Caffeine was first shown to improve exercise performance by Costill et al. (1978),

Corresponding Author: Abbie E. Smith-Ryan, PhD, CSCS*D, Department of Exercise and Sport Science, University of North Carolina-Chapel Hill, 312 Woollen, Chapel Hill, NC 27599, Office: 919-962-2574, Fax: 919-962-6325, abbsmith@email.unc.edu. The content is solely the responsibility of the authors and does not necessarily represent the official views of the NIH. 
who demonstrated an increase in cycling time to exhaustion following coffee consumption. The primary ergogenic mechanism of caffeine is antagonism of adenosine receptors (Goldstein et al., 2010). Caffeine may also affect performance via both central and peripheral mechanisms by altering pain and effort perception (Doherty \& Smith, 2005), calcium kinetics in the sarcoplasmic reticulum (Allen \& Westerblad, 1995; Tallis, James, Cox, \& Duncan, 2012; Weber \& Herz, 1968), and sodium/potassium ATPase pump activity (M. Mohr, Nielsen, \& Bangsbo, 2011), among other potential mechanisms (Goldstein et al., 2010). Caffeine in doses of 3-6 mg/kg bodyweight has been consistently shown to enhance endurance performance (Goldstein et al., 2010), but findings have been equivocal in the context of strength and sprint performance. Evidence currently suggests that caffeine may improve sprint performance in trained subjects (Collomp, Ahmaidi, Chatard, Audran, \& Prefaut, 1992; Juan Del Coso et al., 2012; Lara et al., 2014; Wiles, Coleman, Tegerdine, \& Swaine, 2006; Woolf, Bidwell, \& Carlson, 2008), but not in untrained subjects (Collomp, Ahmaidi, Audran, Chanal, \& Prefaut, 1991; Crowe, Leicht, \& Spinks, 2006; Greer, McLean, \& Graham, 1998; Lorino, Lloyd, Crixell, \& Walker, 2006). Collomp et al. (1992) investigated the effects of caffeine supplementation on sprint performance in both trained and untrained swimmers. By stratifying the sample based on training status, authors showed that sprint velocity was improved only in the highly-trained group of swimmers. This distinction may be related to anaerobic training adaptations, or reduced training variability in trained subjects.

Studies investigating caffeine's effects on strength have reported mixed results. Although Woolf et al. (2008) reported an ergogenic effect of caffeine on chest press endurance, no improvement in leg press endurance was observed. Beck at al. (2006) documented an improvement in bench press one-rep max (1RM), but no improvement in bench press endurance, leg extension 1RM, or leg extension endurance. Subsequent research by Woolf et al. (2009) revealed no significant effect on bench press endurance, and Astorino et al. (2008) did not find any significant improvements in bench press or leg press 1RM or endurance after acute caffeine ingestion. More recent findings have shown caffeine intake to have beneficial effects on repetitions to fatigue (Duncan \& Oxford, 2011; Duncan, Stanley, Parkhouse, Cook, \& Smith, 2013; Hurley, Hatfield, \& Riebe, 2013), subjective feelings of vigor and fatigue (Duncan \& Oxford, 2011), and perceived exertion and muscle pain (Duncan et al., 2013). Conversely, Astorino et al. (2011) found statistically significant improvements in repetitions to fatigue for only one of four exercises tested; it was concluded that the ergogenic effect of acute caffeine intake was of little practical significance and that individual responses to supplementation were variable. It is possible that insufficient sample sizes, variation in individual responses, or differences in caffeine dosing and exercise protocols may partially explain inconsistent outcomes in research to date.

The first study reporting an ergogenic effect of caffeine used coffee as a caffeine source (Costill et al., 1978), but it was later suggested that coffee was less ergogenic than a caffeine-matched dose of caffeine anhydrous (Graham, Hibbert, \& Sathasivam, 1998). Graham et al. (1998) compared the effects of coffee and caffeine anhydrous, and found that only caffeine anhydrous resulted in significant improvements in running time to exhaustion. The authors hypothesized that the differential responses to coffee and caffeine may be attributed to one or more of the numerous biologically active compounds found in coffee. 
One such group of bioactive compounds, known as chlorogenic acids, are present in coffee in varying amounts (Crozier, Stalmach, Lean, \& Crozier, 2012) and may antagonize caffeine's effects on adenosine receptors (de Paulis et al., 2002). Contrary to this hypothesis, McLellan \& Bell (2004) reported that the ergogenic effect of caffeine supplementation was not affected by coffee consumption 30 minutes prior. More recently, Hodgson et al. (2013) showed that energy-based target time trial performance was improved to a similar degree by caffeine-matched doses of coffee and caffeine anhydrous. While Graham et al. (1998) found no significant performance improvement following coffee ingestion, other studies have reported an ergogenic effect using similar doses of coffee before exercise (Costill et al., 1978; Hodgson et al., 2013; Wiles, Bird, Hopkins, \& Riley, 1992). Therefore, the purpose of the current study was to compare the effects of caffeine-matched doses of coffee and caffeine anhydrous on strength and repeated sprint performance. It was hypothesized that caffeine anhydrous ingestion would improve strength and sprint performance to a greater extent than coffee or placebo.

\section{Methods}

\section{Experimental Design}

The current study consisted of two laboratory visits, separated by at least 48 hours. Baseline testing included one-rep maximum (1RM) and repetitions to fatigue (RTF) for leg press (LP) and bench press (BP), and a repeated sprint protocol to determine peak power (PP) and total work output (TW). At least 48 hours after baseline testing, participants returned for a second visit. In a double-blind fashion, participants were randomly assigned, using random allocation software, into one of three equal groups consisting of either $300 \mathrm{mg}$ caffeine anhydrous (CAF), a caffeine-matched dose of coffee (COF), or placebo (PLA). Thirty minutes after ingestion, exercise testing was repeated. All methodology was approved by the University's Biomedical Institutional Review Board, and all participants signed an informed consent prior to participation.

\section{Subjects}

Fifty-six male participants enrolled in the current study. Fifty-four participants (Mean \pm SD; Age $=20.1 \pm 2.1 \mathrm{yrs} ;$ Stature $=177.3 \pm 5.6 \mathrm{~cm}$; Body mass $=78.8 \pm 8.8 \mathrm{~kg}$ ) completed the study, as two participants dropped out after baseline testing for personal reasons. All participants were engaged in resistance training for at least 30 minutes a day, three days a week, for at least three months prior to the study, and weighed between $60-100 \mathrm{~kg}$. Participants were excluded if they had supplemented with creatine or beta-alanine in the three months prior to the study. Participants were also excluded if dietary analysis or prescreening questionnaires revealed habitual caffeine intake of $>800 \mathrm{mg}$ per day, a history of sensitivity or adverse reactions to caffeine consumption, or regular consumption of any food or drug in a quantity that may significantly influence caffeine metabolism, such as grapefruit juice, cigarette smoke, and a number of pharmaceutical drugs. Participants were educated on various foods and beverages containing caffeine and asked to list their normal weekly intake of such products, which was used to calculate habitual caffeine intake for the sample (32.9 $\pm 59.6 \mathrm{mg} /$ day; $0.42 \mathrm{mg} / \mathrm{kg} /$ day). Participants were encouraged to maintain normal diet and exercise habits throughout the duration of the study, but were asked to abstain from caffeine 
intake for at least 48 hours prior to all exercise testing and abstain from vigorous exercise for at least 24 hours prior to testing. Participants completed a three-day diet log, which was analyzed using The Food Processor software (ESHA Research, Salem, OR, USA) to evaluate habitual dietary intakes. Exercise testing sessions took place at a similar time of day ( $\pm 2 \mathrm{hrs}$ ), and participants were instructed to replicate similar food intake before all lab visits. Descriptive characteristics and dietary intakes are listed by group in Table 1. No values were significantly different ( $p>0.05$ ) between groups at baseline.

\section{One Rep Max (1RM) \& Repetitions to Fatigue (RTF)}

Participants completed a 1RM strength test, for LP followed by BP, using free weights and a spotter. To begin the warm up, each participant performed a set of 8-10 repetitions, with a weight that was approximately $50 \%$ of the predicted 1RM. Participants rested for 1-2 minutes, after which a set of 4-6 repetitions was completed with a load of approximately $80 \%$ of the predicted 1RM. After a 2-minute rest period, the weight was increased to an estimated 1RM load, and the participants attempted a single repetition with the weight. After the completion of each successful 1RM attempt, the weight was increased until failure was reached, with 2-3 minutes of rest between 1RM attempts. Once the 1RM was determined, participants were given a three minute rest period before the RTF test, in which participants lifted $80 \%$ of the 1RM load for as many continuous repetitions as possible in a single set, until a full repetition could no longer be completed with appropriate form. For subsequent post-testing, the load used for RTF tests remained constant (80\% of baseline 1RM). For all testing sessions, 1RM and RTF testing for LP preceded BP. Reliability calculations from this laboratory indicate an intraclass correlation coefficient $\left(\mathrm{ICC}_{2,1}\right)$ of 0.97 and 0.99 for LP and BP 1RM, respectively. The standard error of measurement (SEM) for LP and BP 1RM are $7.4 \mathrm{~kg}$ and $1.9 \mathrm{~kg}$, respectively.

\section{Repeated Sprint Protocol}

Participants completed a repeated sprint test modified from the protocol described by Wiroth et al. (2001) ten minutes after the conclusion of strength testing. The test began with a selfpaced five minute warm up with a standardized load of $0.5 \mathrm{~kg}$ on a Monark friction-braked cycle ergometer (Ergomedic 894 E; Monark, Stockholm, Sweden). Following the warm up, participants completed a series of five maximal sprints lasting 10 seconds each, with a load of $95 \mathrm{~g} / \mathrm{kg}$ of body mass. Participants were instructed to remain seated throughout the entire sprint and were verbally encouraged throughout the test. After each sprint, participants were given 60 seconds of passive recovery while seated on the cycle ergometer. For each sprint, PP and TW were evaluated using the default software (Monark ATS Software; Monark, Stockholm, Sweden). For this sprint protocol, reliability calculations from this laboratory indicate an ICC of 0.96 and SEM of 19.7 watts for average peak power.

\section{Supplementation}

Participants were randomly assigned, according to a computer generated allocation sequence, to one of three treatments: $300 \mathrm{mg}$ of caffeine anhydrous (CAF; $3-5 \mathrm{mg} / \mathrm{kg}$ body mass; Smart Powders, Graham, NC, USA) with noncaloric flavoring (Crystal Light; Kraft Foods, Northfield, IL, USA), $8.9 \mathrm{~g}$ of dehydrated coffee (COF; yields $303 \mathrm{mg}$ caffeine [3-5 $\mathrm{mg} / \mathrm{kg}$ body mass]; Nescafé House Blend; Nestle, Vevey, Switzerland), or a placebo 
beverage with noncaloric flavoring (3.8 g Crystal Light). Participants were required to weigh between $60-100 \mathrm{~kg}$ to ensure that the flat $300 \mathrm{mg}$ caffeine dose provided a relative caffeine dose of $3-5 \mathrm{mg} / \mathrm{kg}$ body mass. Based on the average weight of each group, CAF received a relative caffeine dose of $3.85 \mathrm{mg} / \mathrm{kg}$, and COF received $3.84 \mathrm{mg} / \mathrm{kg}$. Blinding was completed by a member of the research staff that did not administer treatments. Supplements were mixed by research staff, with $350 \mathrm{~mL}$ of water, in opaque containers so neither participants nor investigators knew which treatment was being given. Supplements were consumed 30 minutes prior to exercise testing. Due to the distinct coffee taste, this was considered a partially blinded study, although subjects were not aware if the coffee-flavored beverage was caffeinated or not. Previous research using a similar product verified a caffeine concentration of $3.4 \mathrm{~g}$ caffeine/100 $\mathrm{g}$ of instant coffee (Hodgson et al., 2013). As such, caffeine content was matched between CAF and COF (300 mg and $303 \mathrm{mg}$, respectively).

\section{Statistical Analysis}

Data are expressed as mean \pm standard deviation. A series of one-way ANOVAs were used to evaluate baseline differences between groups for dietary intakes and strength and sprint outcomes. For strength outcomes (1RM and RTF for LP and BP), change scores were calculated from baseline testing to post-testing. Four separate one-way ANOVAs were used to compare change scores between groups; in the event of a significant interaction, post hoc comparisons were analyzed using the Bonferroni method. When baseline values were significantly different between groups (LP RTF), change scores were analyzed using a oneway ANCOVA, covaried for baseline values. In addition, 95\% confidence intervals [mean $\pm 1.96 \times$ standard error of the mean (SEM)] were constructed for all strength outcomes. When the $95 \%$ confidence interval did not include zero, the change was considered significant ( $\mathrm{p} \unlhd(05)$.

For each sprint, change scores from baseline to post-testing were calculated for both PP and TW. A series of two-way $(3 \times 5$; treatment $\times$ sprint $)$ mixed factorial ANOVAs were used to compare change scores between treatments for each sprint. The Bonferroni method was used to evaluate post hoc comparisons. Peak power and TW values were averaged between all five sprints to calculate average PP and TW for the entire sprint protocol, and change scores were calculated for these values. Measures of PP and TW were also evaluated using 95\% confidence intervals. Analyses were performed using SPSS software (Version 20.0; IBM, Armonk, NY, USA), and 95\% confidence intervals were calculated and plotted in Microsoft Excel (Version 2011, Microsoft Corporation; The Microsoft Network, LLC, Richmond, WA, USA). Statistical significance was set a priori at a $₫ \mathbf{0 . 0 5}$.

\section{Results}

\section{Strength outcomes}

There were no significant interaction effects for BP $1 \mathrm{RM}$ ( $\mathrm{p}=0.78$; Table 2) or BP RTF $(\mathrm{p}=0.47$; Figure 1$)$, indicating no difference in change scores between treatments. Only LP RTF was significantly different between groups at baseline ( $\mathrm{p}=0.04)$. When covaried for baseline differences, change scores for LP RTF were not significantly different between groups ( $\mathrm{p}=0.49$; Figure 1). There was a significant interaction effect for LP $1 \mathrm{RM}(\mathrm{p}=0.03)$; 
post hoc comparisons revealed a significantly greater improvement in LP 1RM for COF compared to CAF ( $\mathrm{p}=0.04)$, with no differences between COF and PLA ( $\mathrm{p}=0.99)$ or CAF and PLA ( $\mathrm{p}=0.10$; Table 2).

Analysis of 95\% confidence intervals of change scores revealed significant improvements for all groups in all strength measures (Figure 1; Table 2), with significantly greater improvements in LP 1RM for COF [CI: 10.7, $18.5 \mathrm{~kg}$ ] in comparison to CAF [3.3, 10.6 kg].

\section{Sprint outcomes}

For changes in PP, no sprint $\times$ treatment interaction was observed $(\mathrm{p}=0.17)$, with no main effects for sprint $(\mathrm{p}=0.24)$ or treatment $(\mathrm{p}=0.25)$. For changes in $\mathrm{TW}$, no sprint $\times$ treatment interaction was observed $(\mathrm{p}=0.15)$. There was no main effect for treatment $(\mathrm{p}=0.16)$, but there was a significant main effect for sprint $(\mathrm{p}=0.02)$. Post hoc analyses showed a reduction in TW from Sprint 1 to Sprint 3 ( $\mathrm{p}=0.03$ ), with a nonsignificant TW reduction from Sprint 1 to Sprint $4(\mathrm{p}=0.06)$.

Analysis of $95 \%$ confidence intervals revealed a significant increase in Sprint 1 TW for CAF [CI: 81.4, 623.9 joules (J)], but not for COF or PLA (Figure 2). Sprint 4 PP was significantly reduced in PLA [CI: $-64.9,-2.5$ watts (W)], but not in COF $[-34.4,36.6 \mathrm{~W}]$ or CAF [-42.8, 22.2 W]. Total work was significantly reduced in Sprint 2 [CI: $-321.2,-66.1 \mathrm{~J}]$ and Sprint $4[-403.1,-57.6 \mathrm{~J}]$ for PLA, but not for COF [-19.5, 439.3 J and $-291.2,118.3 \mathrm{~J}]$ or CAF $[-415.8,103.3 \mathrm{~J}$ and $-300.9,129.5 \mathrm{~J}]$. Further, average TW was reduced in PLA [CI: $-219.0,-40.2 \mathrm{~J}]$, but not in COF or CAF (Figure 2).

\section{Discussion}

A number of studies have demonstrated that CAF supplementation improves endurance exercise performance (Goldstein et al., 2010). Far less research has investigated its effects on strength and sprint performance, and to our knowledge CAF and COF have not been directly compared in this context. Results of the current study revealed that neither CAF nor COF resulted in significantly greater 1RM or RTF outcomes than PLA for bench press or leg press. Analysis of $95 \%$ confidence intervals suggests an ergogenic effect of both CAF and COF during a repeated sprint protocol. Sprint $1 \mathrm{TW}$ was significantly improved in CAF only. Further, PLA experienced reductions in sprint 4 PP, sprints 2 and 4 TW, and average $\mathrm{TW}$, while these reductions were attenuated by both CAF and COF. The current results may have implications for anaerobic athletes seeking to improve repeated sprint performance with pre-exercise CAF or COF ingestion.

In the current study, BP 1RM, BP RTF, and LP RTF improved in all groups, with no significant differences between treatments. Despite the recruitment of resistance-trained participants, improvement in the PLA group may indicate that a learning or placebo effect led to improved performance. Interestingly, no such effect was observed for the cycling test, which was likely a more unfamiliar exercise test based on inclusion criteria. Coffee improved LP 1RM more than CAF, but not more than PLA. Previous CAF studies investigating similar strength measurements have reported equivocal results. Beck et al. (2006) demonstrated that pre-exercise CAF supplementation increased BP 1RM, but did not 
affect BP endurance, leg extension 1RM, or leg extension endurance. Conversely, Woolf et al. (2008) found an improvement in chest press endurance, but not LP endurance. Other studies by Woolf et al. (2009) and Astorino et al. (2008) found no effect on BP endurance, and no effect BP or LP 1RM or endurance, respectively. Graham et al. (1998) previously indicated that $\mathrm{CAF}$ improved endurance performance more than a caffeine-matched dose of COF; more recent research has failed to support these findings (Hodgson et al., 2013). To our knowledge, the current study is the first to investigate this question in the context of strength and sprint performance, and results do not support the hypothesis that CAF is superior to COF for performance. Coffee improved LP 1RM more than CAF, but the underlying mechanism for this difference is not yet known. Previous research has indicated that individual responses to CAF can vary (Goldstein et al., 2010), and it is therefore possible that individuals in the CAF group reacted less favorably to caffeine than the COF group. Although large standard deviations suggest appreciable inter-individual differences in self-reported habitual daily caffeine intake, average intake was similar between CAF and COF groups $(41.0 \pm 64.5 \mathrm{mg}$ vs. $33.3 \pm 59.4 \mathrm{mg}$, respectively), and participants abstained from caffeinated products for a minimum of 48 hours prior to all testing sessions to eliminate short-term habituation. More research is needed to investigate this finding, but these results do not collectively demonstrate a consistent difference in strength outcomes between acute CAF, COF, or PLA ingestion.

In the current study, sprint $1 \mathrm{TW}$ was significantly improved by CAF, but not by COF or PLA. Further, the PLA group experienced reductions in PP and TW during post-testing, while $\mathrm{PP}$ and TW were maintained with $\mathrm{CAF}$ or COF. These results indicate that both COF and CAF had an ergogenic effect on the repeated sprint test, with no clear difference between the two groups. These findings are consistent with previous research documenting improvements for both single (Woolf et al., 2008) and repeated sprint performance (Schneiker, Bishop, Dawson, \& Hackett, 2006) in trained subjects with CAF use, and suggest that improvements are similar with COF. In the context of high-intensity sprints, the ergogenic effects of caffeine are most likely related to its effects on adenosine receptors (Goldstein et al., 2010) and pain/effort perception (Doherty \& Smith, 2005). Participants in a previous study reported reduced leg pain during cycling to exhaustion at $60 \%$ of $\mathrm{VO}_{2}$ peak following caffeine ingestion (Cramer, 2008). While leg pain was not directly assessed in the current study, attenuation of pain or effort perception would likely be advantageous in the strenuous sprint protocol employed. Additionally, caffeine's effects on cycling performance may relate to direct effects of caffeine on muscle. Electronically stimulated cycling time to exhaustion was improved by caffeine in a sample of paraplegic and tetraplegic participants (T. Mohr, Van Soeren, Graham, \& Kjaer, 1998). Results indicated that this effect was not influenced by catecholamines, substrate utilization, or central factors, and was possibly attributable to peripheral effects on excitation-contraction coupling. Power reductions are not likely related to caffeine withdrawal, as habitual caffeine intake was low in the PLA group ( $24.2 \pm 56.8 \mathrm{mg} /$ day), and participants observed a standardized, 48-hour abstention from caffeinated products prior to baseline testing. While baseline testing and post-testing were separated by at least 48 hours, power reductions in the PLA group may relate to the strenuous nature of the testing session, or residual soreness or fatigue from pre-testing. These power reductions were attenuated by both $\mathrm{CAF}$ and $\mathrm{COF}$, suggesting that $\mathrm{CAF}$ and 
COF may be beneficial for athletes, who typically complete multiple strenuous training sessions within a given week.

Previous research has suggested that one or more of the bioactive compounds in COF, such as chlorogenic acids, may attenuate the ergogenic effect of caffeine (Goldstein et al., 2010; Graham et al., 1998). In agreement with more recent research (Hodgson et al., 2013; McLellan \& Bell, 2004), results of the current study suggest that COF and CAF yield similar benefits for high-intensity exercise. While caffeine has previously been associated with negative health outcomes, recent research has suggested that habitual COF consumption may be beneficial after controlling for confounding variables such as smoking and heavy alcohol consumption (Freedman, Park, Abnet, Hollenbeck, \& Sinha, 2012; Gonzalez de Mejia \& Ramirez-Mares, 2014). Coffee contains numerous biologically active compounds, and authors have proposed that some of these compounds may counteract the negative health effects associated with caffeine intake (Gonzalez de Mejia \& Ramirez-Mares, 2014). As such, COF may be considered a suitable source of caffeine prior to high-intensity exercise, with results of the current study suggesting a similar ergogenic effect when compared to CAF.

A limitation of the current study is the provision of a flat dose of caffeine $(300 \mathrm{mg}$ ) rather than scaling the dosage to bodyweight. Previous studies have demonstrated that 3 or $6 \mathrm{mg} / \mathrm{kg}$ doses of caffeine improve endurance performance to a similar degree (Graham \& Spriet, 1995), and that a $3 \mathrm{mg} / \mathrm{kg}$ dose is sufficient to improve strength outcomes similar to those measured in the current study (Del Coso, Salinero, Gonzalez-Millan, Abian-Vicen, \& PerezGonzalez, 2012). Further, the ergogenic effects of caffeine supplementation do not generally follow a dose-response relationship (Graham \& Spriet, 1995; Pasman, van Baak, Jeukendrup, \& de Haan, 1995). Subjects in the current study reported low habitual caffeine intake $(32.9 \pm 59.6 \mathrm{mg} / \mathrm{day})$, which may limit generalizability to heavy caffeine users. Although previous studies have suggested that changes in graded exercise performance (Dodd, Brooks, Powers, \& Tulley, 1991), repeated sprint performance (Glaister et al., 2008), and stimulated muscle force (Tarnopolsky \& Cupido, 2000) do not differ because caffeinehabituated and caffeine-naïve subjects, more research on caffeine habituation is warranted. Many caffeine studies provide supplements one hour prior to exercise, whereas supplements in the current study were ingested 30 minutes prior to exercise. This time frame was selected because $90 \%$ of caffeine is cleared from the stomach within 20 minutes of ingestion, with peak values occurring as early as 30-45 minutes after ingestion (Gonzalez de Mejia \& Ramirez-Mares, 2014), and because the exercise test protocol took roughly one hour to complete. Despite the recruitment of resistance-trained participants, it appeared that a learning effect or a placebo effect was observed for strength outcomes, but not for sprint outcomes. Finally, reductions in power output in the PLA group may suggest that CAF and $\mathrm{COF}$ attenuated fatigue throughout the series of exercise tests in post-testing. These power reductions could also indicate that participants were not fully recovered between pre- and post-testing sessions, which were separated by at least 48 hours. If so, attenuation of power decrements may reveal a valuable effect of $\mathrm{CAF}$ and $\mathrm{COF}$ for athletes completing multiple strenuous exercise bouts in the same week. While delayed onset muscle soreness (DOMS) generally peaks 24-72 hours after exercise (Cheung, Hume, \& Maxwell, 2003), the average time between visits was similar in all groups and beyond the range for peak soreness $(\mathrm{CAF}=$ 
6.1 days, $\mathrm{COF}=5.3, \mathrm{PLA}=5.2$ ). These values suggest that DOMS was not likely to confound between-group comparisons in the current study. Future research should seek to determine optimal dosing strategies for $\mathrm{COF}$ and $\mathrm{CAF}$ prior to high-intensity exercise.

\section{Conclusion}

The current study demonstrated an ergogenic effect of caffeine-matched doses of CAF and $\mathrm{COF}$ on repeated sprint exercise, with no clear difference between treatments. Neither CAF nor COF improved strength outcomes compared to placebo. In trained males, caffeine dosed at $3-5 \mathrm{mg} / \mathrm{kg}$ may improve high-intensity sprint performance when ingested 30 minutes prior to exercise. Caffeine anhydrous does not appear to improve performance more than coffee. Given the health benefits of habitual coffee consumption (Gonzalez de Mejia \& Ramirez-Mares, 2014), coffee appears to be a suitable source of caffeine to improve sprint performance, particularly when multiple strenuous bouts are completed in the same week.

\section{Acknowledgments}

This study was funded by the National Strength and Conditioning Association Foundation. The project described was also supported by the National Center for Advancing Translational Sciences, National Institutes of Health, through Grant 1KL2TR001109.

\section{References}

Allen DG, Westerblad H. The effects of caffeine on intracellular calcium, force and the rate of relaxation of mouse skeletal muscle. Journal of Physiology. 1995; 487(Pt 2):331-342. [PubMed: 8558467]

Astorino TA, Martin BJ, Schachtsiek L, Wong K, Ng K. Minimal effect of acute caffeine ingestion on intense resistance training performance. Journal of Strength and Conditioning Research. 2011; 25(6):1752-1758. DOI: 10.1519/JSC.0b013e3181ddf6db [PubMed: 21293304]

Astorino TA, Rohmann RL, Firth K. Effect of caffeine ingestion on one-repetition maximum muscular strength. European Journal of Applied Physiology. 2008; 102(2):127-132. DOI: 10.1007/ s00421-007-0557-x [PubMed: 17851681]

Barone JJ, Roberts HR. Caffeine consumption. Food and Chemical Toxicology. 1996; 34(1):119-129. [PubMed: 8603790]

Beck TW, Housh TJ, Schmidt RJ, Johnson GO, Housh DJ, Coburn JW, Malek MH. The acute effects of a caffeine-containing supplement on strength, muscular endurance, and anaerobic capabilities. Journal of Strength and Conditioning Research. 2006; 20(3):506-510. DOI: 10.1519/18285.1 [PubMed: 16937961]

Cheung K, Hume P, Maxwell L. Delayed onset muscle soreness : treatment strategies and performance factors. Sports Medicine. 2003; 33(2):145-164. [PubMed: 12617692]

Collomp K, Ahmaidi S, Audran M, Chanal JL, Prefaut C. Effects of caffeine ingestion on performance and anaerobic metabolism during the Wingate Test. International Journal of Sports Medicine. 1991; 12(5):439-443. DOI: 10.1055/s-2007-1024710 [PubMed: 1752708]

Collomp K, Ahmaidi S, Chatard JC, Audran M, Prefaut C. Benefits of caffeine ingestion on sprint performance in trained and untrained swimmers. European Journal of Applied Physiology and Occupational Physiology. 1992; 64(4):377-380. [PubMed: 1592065]

Costill DL, Dalsky GP, Fink WJ. Effects of caffeine ingestion on metabolism and exercise performance. Medicine and Science in Sports. 1978; 10(3):155-158. [PubMed: 723503]

Cramer, JT. Creatine Supplementation in Endurance Sports. In: Stout, JR.; Antonio, J.; Kalman, D., editors. Essentials of Creatine in Sports and Health. Humana Press; 2008. p. 45-99. 
Crowe MJ, Leicht AS, Spinks WL. Physiological and cognitive responses to caffeine during repeated, high-intensity exercise. International Journal of Sport Nutrition and Exercise Metabolism. 2006; 16(5):528-544. [PubMed: 17240784]

Crozier TW, Stalmach A, Lean ME, Crozier A. Espresso coffees, caffeine and chlorogenic acid intake: potential health implications. Food \& Function. 2012; 3(1):30-33. DOI: 10.1039/c1fo10240k [PubMed: 22130653]

de Paulis T, Schmidt DE, Bruchey AK, Kirby MT, McDonald MP, Commers P, Martin PR. Dicinnamoylquinides in roasted coffee inhibit the human adenosine transporter. European Journal of Pharmacology. 2002; 442(3):215-223. [PubMed: 12065074]

Del Coso J, Muñoz-Fernández VE, Muñoz G, Fernández-Elías VE, Ortega JF, Hamouti N, MuñozGuerra J. Effects of a Caffeine-Containing Energy Drink on Simulated Soccer Performance. PloS One. 2012; 7(2):e31380.doi: 10.1371/journal.pone.0031380 [PubMed: 22348079]

Del Coso J, Salinero JJ, Gonzalez-Millan C, Abian-Vicen J, Perez-Gonzalez B. Dose response effects of a caffeine-containing energy drink on muscle performance: a repeated measures design. Journal of the International Society of Sports Nutrition. 2012; 9(1):21.doi: 10.1186/1550-2783-9-21 [PubMed: 22569090]

Dodd SL, Brooks E, Powers SK, Tulley R. The effects of caffeine on graded exercise performance in caffeine naive versus habituated subjects. European Journal of Applied Physiology and Occupational Physiology. 1991; 62(6):424-429. [PubMed: 1893906]

Doherty M, Smith PM. Effects of caffeine ingestion on rating of perceived exertion during and after exercise: a meta-analysis. Scandinavian Journal of Medicine and Science in Sports. 2005; 15(2): 69-78. DOI: 10.1111/j.1600-0838.2005.00445.x [PubMed: 15773860]

Duncan MJ, Oxford SW. The effect of caffeine ingestion on mood state and bench press performance to failure. Journal of Strength and Conditioning Research. 2011; 25(1):178-185. DOI: 10.1519/ JSC.0b013e318201bddb [PubMed: 21157384]

Duncan MJ, Stanley M, Parkhouse N, Cook K, Smith M. Acute caffeine ingestion enhances strength performance and reduces perceived exertion and muscle pain perception during resistance exercise. European Journal of Sport Science. 2013; 13(4):392-399. DOI: 10.1080/17461391.2011.635811 [PubMed: 23834545]

Freedman ND, Park Y, Abnet CC, Hollenbeck AR, Sinha R. Association of coffee drinking with total and cause-specific mortality. New England Journal of Medicine. 2012; 366(20):1891-1904. DOI: 10.1056/NEJMoa1112010 [PubMed: 22591295]

Glaister M, Howatson G, Abraham CS, Lockey RA, Goodwin JE, Foley P, McInnes G. Caffeine supplementation and multiple sprint running performance. Medicine and Science in Sports and Exercise. 2008; 40(10):1835-1840. DOI: 10.1249/MSS.0b013e31817a8ad2 [PubMed: 18799995]

Goldstein ER, Ziegenfuss T, Kalman D, Kreider R, Campbell B, Wilborn C, Antonio J. International society of sports nutrition position stand: caffeine and performance. Journal of the International Society of Sports Nutrition. 2010; 7(1):5.doi: 10.1186/1550-2783-7-5 [PubMed: 20205813]

Gonzalez de Mejia E, Ramirez-Mares MV. Impact of caffeine and coffee on our health. Trends in Endocrinology and Metabolism. 2014; 25(10):489-492. DOI: 10.1016/j.tem.2014.07.003 [PubMed: 25124982]

Graham TE, Hibbert E, Sathasivam P. Metabolic and exercise endurance effects of coffee and caffeine ingestion. Journal of Applied Physiology (1985). 1998; 85(3):883-889.

Graham TE, Spriet LL. Metabolic, catecholamine, and exercise performance responses to various doses of caffeine. Journal of Applied Physiology (1985). 1995; 78(3):867-874.

Greer F, McLean C, Graham TE. Caffeine, performance, and metabolism during repeated Wingate exercise tests. Journal of Applied Physiology (1985). 1998; 85(4):1502-1508.

Hodgson AB, Randell RK, Jeukendrup AE. The metabolic and performance effects of caffeine compared to coffee during endurance exercise. PloS One. 2013; 8(4):e59561.doi: 10.1371/ journal.pone.0059561 [PubMed: 23573201]

Hurley CF, Hatfield DL, Riebe DA. The effect of caffeine ingestion on delayed onset muscle soreness. Journal of Strength and Conditioning Research. 2013; 27(11):3101-3109. DOI: 10.1519/JSC. 0b013e3182a99477 [PubMed: 24164961] 
Lara B, Gonzalez-Millan C, Salinero JJ, Abian-Vicen J, Areces F, Barbero-Alvarez JC, Del Coso J. Caffeine-containing energy drink improves physical performance in female soccer players. Amino Acids. 2014; 46(5):1385-1392. DOI: 10.1007/s00726-014-1709-z [PubMed: 24615239]

Lorino AJ, Lloyd LK, Crixell SH, Walker JL. The effects of caffeine on athletic agility. Journal of Strength and Conditioning Research. 2006; 20(4):851-854. DOI: 10.1519/R-17445.1 [PubMed: 17194233]

McLellan TM, Bell DG. The impact of prior coffee consumption on the subsequent ergogenic effect of anhydrous caffeine. International Journal of Sport Nutrition and Exercise Metabolism. 2004; 14(6):698-708. [PubMed: 15657474]

Mohr M, Nielsen JJ, Bangsbo J. Caffeine intake improves intense intermittent exercise performance and reduces muscle interstitial potassium accumulation. Journal of Applied Physiology (1985). 2011; 111(5):1372-1379. DOI: 10.1152/japplphysiol.01028.2010

Mohr T, Van Soeren M, Graham TE, Kjaer M. Caffeine ingestion and metabolic responses of tetraplegic humans during electrical cycling. Journal of Applied Physiology (1985). 1998; 85(3): 979-985.

Pasman WJ, van Baak MA, Jeukendrup AE, de Haan A. The effect of different dosages of caffeine on endurance performance time. International Journal of Sports Medicine. 1995; 16(4):225-230. DOI: 10.1055/s-2007-972996 [PubMed: 7657415]

Schneiker KT, Bishop D, Dawson B, Hackett LP. Effects of caffeine on prolonged intermittent-sprint ability in team-sport athletes. Medicine and Science in Sports and Exercise. 2006; 38(3):578-585. DOI: 10.1249/01.mss.0000188449.18968.62 [PubMed: 16540848]

Tallis J, James RS, Cox VM, Duncan MJ. The effect of physiological concentrations of caffeine on the power output of maximally and submaximally stimulated mouse EDL (fast) and soleus (slow) muscle. Journal of Applied Physiology (1985). 2012; 112(1):64-71. DOI: 10.1152/japplphysiol. 00801.2011

Tarnopolsky M, Cupido C. Caffeine potentiates low frequency skeletal muscle force in habitual and nonhabitual caffeine consumers. Journal of Applied Physiology (1985). 2000; 89(5):1719-1724.

Weber A, Herz R. The relationship between caffeine contracture of intact muscle and the effect of caffeine on reticulum. Journal of General Physiology. 1968; 52(5):750-759. [PubMed: 5688082]

Wiles JD, Bird SR, Hopkins J, Riley M. Effect of caffeinated coffee on running speed, respiratory factors, blood lactate and perceived exertion during 1500-m treadmill running. British Journal of Sports Medicine. 1992; 26(2):116-120. [PubMed: 1623356]

Wiles JD, Coleman D, Tegerdine M, Swaine IL. The effects of caffeine ingestion on performance time, speed and power during a laboratory-based $1 \mathrm{~km}$ cycling time-trial. Journal of Sports Sciences. 2006; 24(11):1165-1171. DOI: 10.1080/02640410500457687 [PubMed: 17035165]

Wiroth JB, Bermon S, Andrei S, Dalloz E, Hebuterne X, Dolisi C. Effects of oral creatine supplementation on maximal pedalling performance in older adults. European Journal of Applied Physiology. 2001; 84(6):533-539. [PubMed: 11482548]

Woolf K, Bidwell WK, Carlson AG. The effect of caffeine as an ergogenic aid in anaerobic exercise. International Journal of Sport Nutrition and Exercise Metabolism. 2008; 18(4):412-429. [PubMed: 18708685]

Woolf K, Bidwell WK, Carlson AG. Effect of caffeine as an ergogenic aid during anaerobic exercise performance in caffeine naive collegiate football players. Journal of Strength and Conditioning Research. 2009; 23(5):1363-1369. DOI: 10.1519/JSC.0b013e3181b3393b [PubMed: 19620930] 


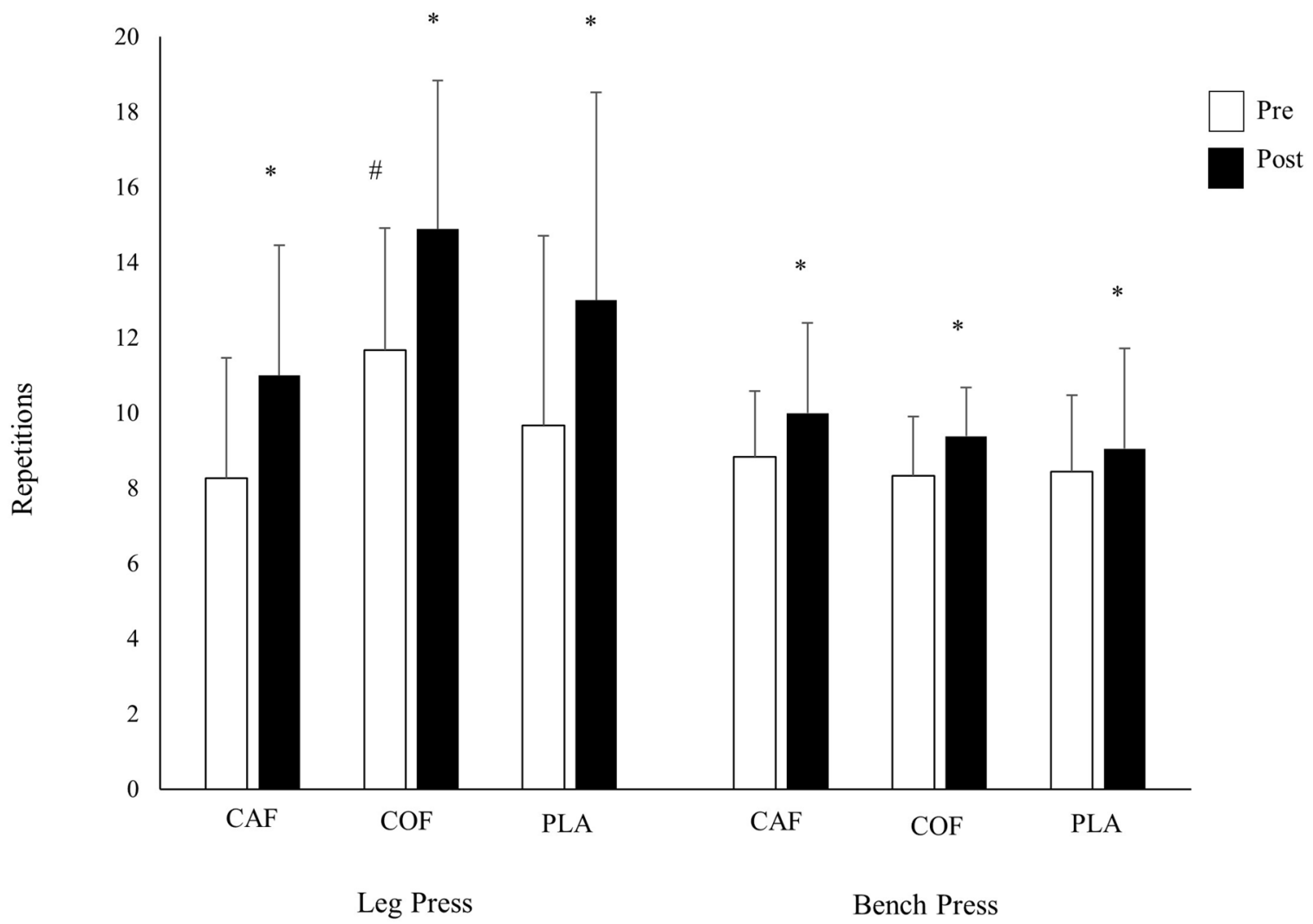

Figure 1.

Effects of supplementation on leg press and bench press repetitions to fatigue. * Significant change from baseline, as determined by $95 \% \mathrm{CI}$; ${ }^{\#}$ significantly greater than $\mathrm{CAF}$ at baseline 


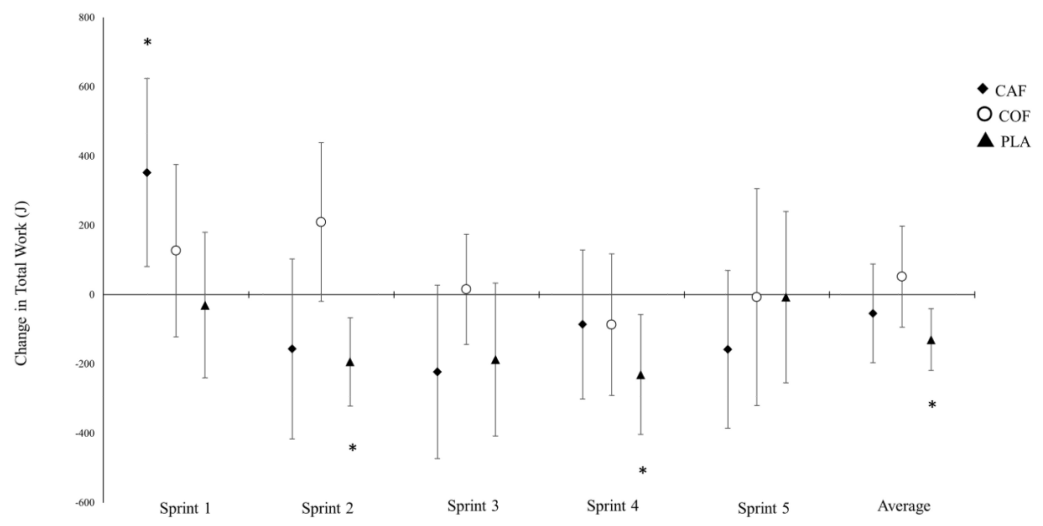

Figure 2.

Changes in total work (TW; joules) after supplementation. *Significant change from baseline, as determined by $95 \% \mathrm{CI}$ 


\section{Table 1}

Baseline characteristics and habitual dietary intakes by group for caffeine (CAF), coffee (COF), and placebo (PLA). Values are mean \pm SD.

\begin{tabular}{lrrr}
\hline & CAF $(\mathbf{n}=\mathbf{1 8})$ & COF $(\mathbf{n}=\mathbf{1 8})$ & PLA $(\mathbf{n}=18)$ \\
\hline Age $(\mathrm{yrs})$ & $20.3 \pm 2.3$ & $20.3 \pm 2.3$ & $19.7 \pm 1.6$ \\
Height $(\mathrm{cm})$ & $178.0 \pm 4.8$ & $176.4 \pm 6.0$ & $177.4 \pm 6.2$ \\
Weight $(\mathrm{kg})$ & $77.9 \pm 7.8$ & $79.0 \pm 10.7$ & $79.4 \pm 8.3$ \\
Calories (kcal) & $2616.2 \pm 745.9$ & $2823.1 \pm 615.8$ & $2514.9 \pm 549.5$ \\
CHO (g/day) & $285.0 \pm 94.0$ & $308.0 \pm 104.0$ & $283.2 \pm 98.1$ \\
FAT (g/day) & $107.6 \pm 43.6$ & $113.7 \pm 32.3$ & $100.4 \pm 29.5$ \\
PRO (g/day) & $129.6 \pm 32.6$ & $142.8 \pm 32.7$ & $125.4 \pm 35.0$ \\
CAF (mg/day) & $41.0 \pm 64.5$ & $33.3 \pm 59.4$ & $24.2 \pm 56.8$ \\
\hline
\end{tabular}

$\mathrm{CHO}=$ carbohydrate intake, $\mathrm{PRO}=$ protein intake, $\mathrm{CAF}=$ habitual caffeine intake 
Table 2

Effects of supplementation on leg press and bench press 1RM $(\mathrm{kg})$

\begin{tabular}{lcccc}
\hline & \multicolumn{2}{c}{ LP 1RM $(\mathbf{k g})$} & \multicolumn{2}{c}{ BP 1RM $(\mathbf{k g})$} \\
\hline Treatment & Pre & Post & Pre & Post \\
\hline CAF & $283.5 \pm 62.9$ & $290.5 \pm 64.1^{*}$ & $93.0 \pm 18.5$ & $94.7 \pm 18.5^{*}$ \\
COF & $309.4 \pm 86.3$ & $324.0 \pm 86.2^{*} \xi$ & $95.5 \pm 17.7$ & $96.7 \pm 18.1^{*}$ \\
PLA & $279.3 \pm 69.1$ & $292.8 \pm 67.5^{*}$ & $86.9 \pm 17.3$ & $88.8 \pm 17.1^{*}$ \\
\hline \multirow{5}{*}{ Significant change from baseline, as determined by 95\%CI } \\
\multirow{5}{*}{ Change score significantly greater than CAF }
\end{tabular}

